\title{
Quantum of electrodynamics theory of high-efficiency excitation energy transfer in laser-driven nanostructure systems*
}

\author{
Dilusha Weeraddana, Malin Premaratne ${ }^{\dagger}$ \\ Advanced Computing and Simulation Laboratory $(A \chi L)$, \\ Department of Electrical and Computer Systems Engineering, \\ Monash University, Clayton, Victoria, 3800, Australia \\ David L. Andrews ${ }^{\ddagger}$ \\ School of Chemistry, University of East Anglia, Norwich Research Park, Norwich NR4 7TJ, United Kingdom
}

(Dated: April 23, 2016)

\begin{abstract}
A fundamental theory is developed for describing laser driven resonance energy transfer (RET) in dimensionally constrained nanostreutures within the framework of quantum electrodynamics. The process of RET communicates electronic excitation between suitably disposed emitter and detector particles in close proximity, activated en excitation of the emitter. Here, we demonstrate that the transfer rate can be significantly increased by propagation of an auxiliary laser beam through a pair of nanostructure particles. This is due to the higher order perturbative contribution to the Förster type RET, in which laser field is applied to stimulate the energy transfer process. We construct a detailed picture of how excitation energy transfer is affected by an off-resonant radiation field, which includes the derivation of second and fourth order quantum amplitudes, and the derivation of-transfer rates; orientational, distance and laser intensity dependences, providing a comprehensive fundamental understanding of laser driven RET in nanostructures. The results of the derivations demonstrate that the geometry of the system exercises considerable control over the laser assisted RET mechanism. Thus, under favourable conformational conditions and relative spacing of donoracceptor nanostructures, auxiliary laser beam is shown to produce an enhancement in the energy migration rate up to $70 \%$.
\end{abstract}

PACS numbers: $31.30 . \mathrm{J}-, 78.70 .-\mathrm{g}, 33.50 . \mathrm{Hv}, 78.67 . \mathrm{De}, 78.67 . \mathrm{Uh}, 78.67 . \mathrm{Hc}$

\section{INTRODUCTION}

In the past few year $\Omega$ miconductor nanowires and quantum dots have developed into advanced, promising classes of materials for numerous next-generation applications in photonics [1-4]. The quantum confinement in these materials make them capable of providing unique and superior optical properties. Thus, nanowires and quantum dots have been extensively exploited in lighting, light harvesting and electronic devices due to strong confinement effects [5-7]. In particular, exciton-exciton interactions through nonradiative resonance energy transfer (RET) of these dimensionally constrained nanostructures have enabled exciting opportunities in light generation and energy harvesting [8-10].

RET gains control across a chemically diverse and extensive range of material systems [11-15]. Energy from the sunlight is captured by photo-biological antenna chromophores, subsequently transferred to a reaction center by a series of hops between other chromophore units with great efficiency by RET $[16,17]$. The phenomenon also has an important function in the operation of organic light-emitting diodes, solar light harvesting and luminescence detectors $[11,18,19]$.

\footnotetext{
† dilusha.weeraddana@monash.edu,malin.premaratne@monash.edu

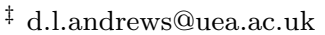

The process of resonance energy transfer was first correctly described by Theodor Förster [20], and is also known as Förster resonance energy transfer (FRET). FRET can be described as the transfer of the excitation energy from an excited donor to a ground-state detector without the process of photon emission/reabsorption. This mechanism of transfer of energy in near-field occurs due to the Coulombic interaction between the resonant transition dipoles of donor and detector species. Furthermore, RET was first discussed in terms of quantum electrodynamics (QED) in pioneering studies by Avery [21], Gomberoff and Power [22]. These studies showed that far-field retardation effects embellish a near-field Coulombic interaction and, radiative and radiationless mechanisms are both necessarily incorporated within a common theory as asymptotic limits. In fact, RET is fully quantum mechanical in nature, and can be comprehensively analysed considering a closed quantum mechanical system where both matter and radiation are quantized [23-26].

Moreover, it has been shown that, electronically excited nanostructures interact with their neighbors differently from their ground-state counterparts $[27,28]$. It also emerges from the use of QED, that a throughput offresonant radiation can also produce significant additional effects on the process of RET between two molecules (Laser Assisted Resonance Energy Transfer) [29]. This is due to coupled absorption and stimulated emission of photons from and into the applied beam. Therefore, following conventional excitation of the donor particle, the 


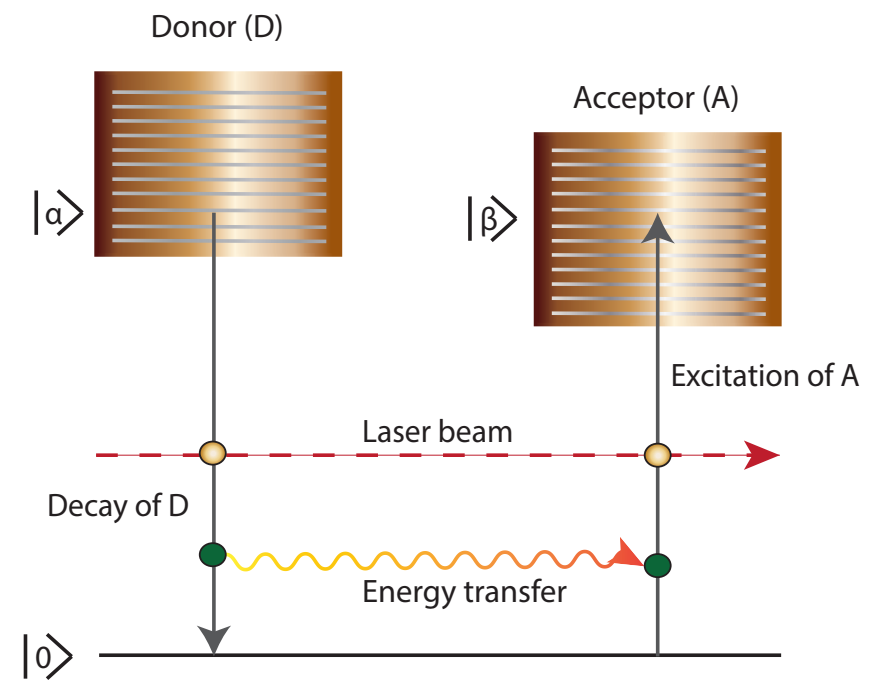

FIG. 1. Schematic depiction of energy transfer from donor to acceptor. Green arrows represent emission and absorption of energy in donor and acceptor particles. Horizontal red dash arrow illustrates the interaction of the laser beam with the donor and acceptor. Horizontal orange wavy arrow represents the energy-transfer from the donor to the acceptor. The Greek letters indicate the relevant electronic excited states and 0 the ground state

transfer rate can be appreciably increased by the propagation of an auxiliary laser beam through the donoracceptor system [30]. A schematic illustration of laser driven RET process is shown in Fig.1.

However, the origin of the complications associated with laser driven RET in nanostructures is deeply rooted in the nature of real and virtual photons which interact with confined geometries. Although the basic principles of RET are well established, there still exist open questions regarding the mechanisms of RET phenomenon in many aspects.

Therefore, the main objective of this paper is to study the effects on the energy transfer process due directly to a laser field impinging on nanowire and quantum dot systems. We propose and model a system consisted of nanowires or quantum dots irradiated by an auxiliary laser beam, by developing a comprehensive quantum electrodynamical analysis. We show that the rate of resonance energy transfer can be greatly increased through an applied off-resonant radiation field under favourable physical configurations of nanostrcutures.

\section{QED PERSPECTIVE OF LASER ASSISTED RESONANCE ENERGY TRANSFER (LARET)}

We review and sharpen the concept of RET based on the quantum theory of light-matter interaction. The key feature of quantum electrodynamics is that both the radiation and matter are subject to quantum mechanical rules. The system Hamiltonian comprises un- perturbed operators for the two matter components, a source, $H_{m a t}^{D}$ and detector, $H_{m a t}^{A}$, two corresponding light-matter interaction terms, $H_{\text {int }}^{D}, H_{\text {int }}^{A}$, and also the second-quantized radiation field $H_{\text {rad }}$;

$$
H_{\text {total }}=H_{m a t}^{D}+H_{m a t}^{A}+H_{i n t}^{D}+H_{i n t}^{A}+H_{\text {rad }} .
$$

Within the electric dipole approximation, the matterfield coupling Hamiltonian $H_{\text {int }}^{\xi}(\xi=D, A)$ is explicitly given by,

$$
H_{i n t}^{\xi}=-\boldsymbol{\mu}(\xi) \cdot \boldsymbol{E}\left(\boldsymbol{R}_{\xi}\right)
$$

where the interaction Hamiltonian compromises contributions for each species $\xi$ located at $\boldsymbol{R}_{\xi}$, the $\boldsymbol{\mu}(\xi)$ is the electric-dipole moment operator and $\boldsymbol{E}\left(\boldsymbol{R}_{\xi}\right)$ is the operator for the electric displacement field at the specified location $\boldsymbol{R}_{\xi}$.

The throughput radiation (with wave vector, $\mathbf{k}$ and polarization, $\lambda$ ) emerges in the final state that is unchanged from its initial state, while the matter system experiences a transfer of energy from $D$ to $A$. Thus, for the initial $\left(\left|\Psi_{I}\right\rangle\right)$ and final $\left(\left|\Psi_{F}\right\rangle\right)$ matter-radiation state vectors are chosen to be the following eigenvectors

$$
\begin{aligned}
& \left|\Psi_{I}\right\rangle=\left|D^{\alpha} A^{0} ; n(\mathbf{k}, \lambda)\right\rangle \\
& \left|\Psi_{F}\right\rangle=\left|D^{0} A^{\beta} ; n(\mathbf{k}, \lambda)\right\rangle
\end{aligned}
$$

Here, superscripts denote donor and acceptor states $\alpha, \beta$.

The quantum probability amplitude or matrix element, $M$, which is expressed through the time-dependent perturbation expansion:

$$
M=\sum_{m=1}^{\infty} M^{(m)}
$$

where $m$ is the number of photonic interactions. For the conventional direct energy transfer, leading contributions to the matrix element are associated with $m=2$, expressive of the two interactions shown in Fig. 2 (a),(b) $[23,30]$, and given by

$$
M^{(2)}=\sum_{R} \frac{\left\langle F\left|H_{i n t}\right| R\right\rangle\left\langle R\left|H_{i n t}\right| I\right\rangle}{E_{I}-E_{R}} .
$$

The effects on the energy-transfer process manifest through interaction with an auxiliary beam, the lowestorder contribution to effect a rate modification will be due to two extra laser-particle interactions. As depicted in Figs. 2 (c), (d), (e), (f), depending on how the throughput radiation interacts with the emitter-detector system, a number of possible laser modified energy transfer mechanisms emerge. Each entails real photon absorption and emission, coupled by a virtual photon mediator. Therefore, the quantum amplitude which accounts for 


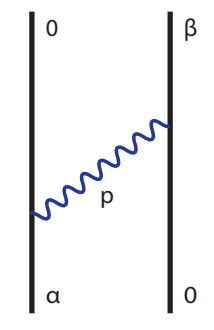

D

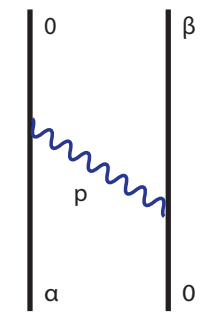

D $\beta$
0
0

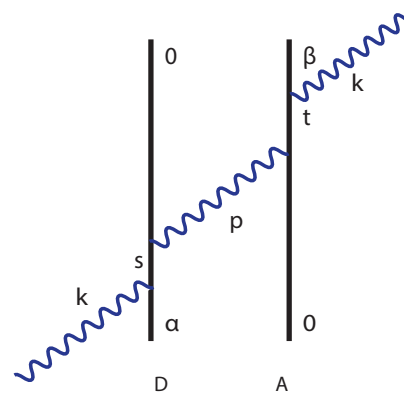

(c) (a)

(b)

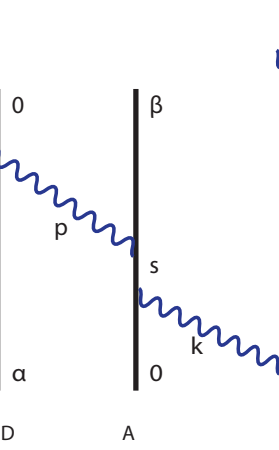

(d)

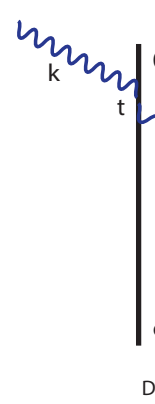

(d)

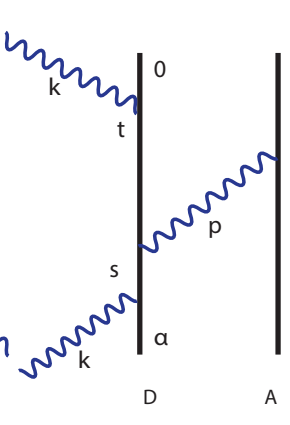

(e)

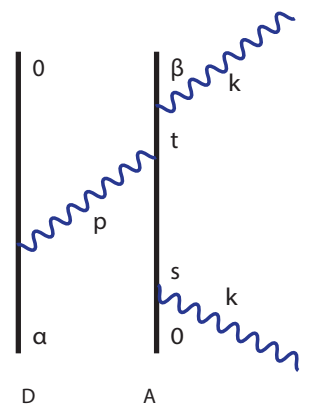

(f)

FIG. 2. Time-ordered Feynman diagrams representing resonance energy transfer between a donor $D$ and acceptor $A$. On each matter vertical line, Greek symbols identify particle electronic excited states, with 0 the corresponding ground state and $r, s$ are intermediate matter states. The transfer is mediated by the virtual photon with wave vector $\boldsymbol{p}_{\text {- }}$ (a),(b) illustrate Feynman diagrams for direct RET- (c) one of 24 time orderings representing one type of laser modified process where both donor and acceptor interact with the auxiliary beam (with wave vector $\boldsymbol{k}$ )- (d) the mirror case of (c)= (e) one of 24 time orderings representative of LARET interactions where only the donor $D$ interacts with the laser beam- (f) photons are absorbed from and emitted back into the laser beam at the acceptor $A$.

these corrections is a fourth-order perturbational theory summing over three intermediate states $R, S$, and $T$.

$M^{(4)}=\sum_{R, S, T} \frac{\left\langle F\left|H_{i n t}\right| T\right\rangle\left\langle T\left|H_{i n t}\right| S\right\rangle\left\langle S\left|H_{i n t}\right| R\right\rangle\left\langle R\left|H_{i n t}\right| I\right\rangle}{\left(E_{I}-E_{R}\right)\left(E_{I}-E_{S}\right)\left(E_{I}-E_{T}\right)}$

Finally, the energy-transfer process modified by the laser field impinging on a donor-acceptor system, can be deduced using Fermi's golden rule [31].

$$
\Gamma_{\text {laser }}^{\text {tot }}=\frac{2 \pi}{\hbar}\left|\sum_{m=1}^{\infty} M^{2 m}\right|^{2} \rho
$$

where $\rho$ is the density of final molecular states of the acceptor particle. The even constraint on the value of $m$ is a result of the nature of LARET. This is because, every energy transfer entails at least two photonic interactions and, in order for the auxiliary laser field to remain unperturbed overall, each matter-field photonic annihilation needs to be twinned with a creation and vice versa. Here, only the second and fourth orders of the perturbation are sufficient as the series rapidly converges for $m \geq 3$.

\section{ENHANCEMENT OF RESONANCE ENERGY TRANSFER BY AN AUXILIARY LASER BEAM}

We explore the effects on the process of energy transfer due directly to a laser beam impinging on a donoracceptor nanostructure system consisting of nanowires and quantum dots. We shall concentrate exclusively on the dominant near-field regime, where the compelling photo-physical mechanism, RET gains control.

\section{A. Nanowire System}

Nanowires are specific type of nanostructures with large aspect ratios and small diameters, and have been the focus of extensive research during the last few decades $[32,33]$. Their length is sufficiently large for easy manipulation as building blocks in fabricating superstructures. Here, we consider the process of resonance energy transfer in a system consisted of NWs illuminated by an auxiliary laser field as illustrated in Fig. (3). Owing to the cylindrical symmetry of NWs, it is convenient to model EM waves using Hankel function of order $n$ [34-36]. Directly substituting into Eq. (6), performing contour in- 


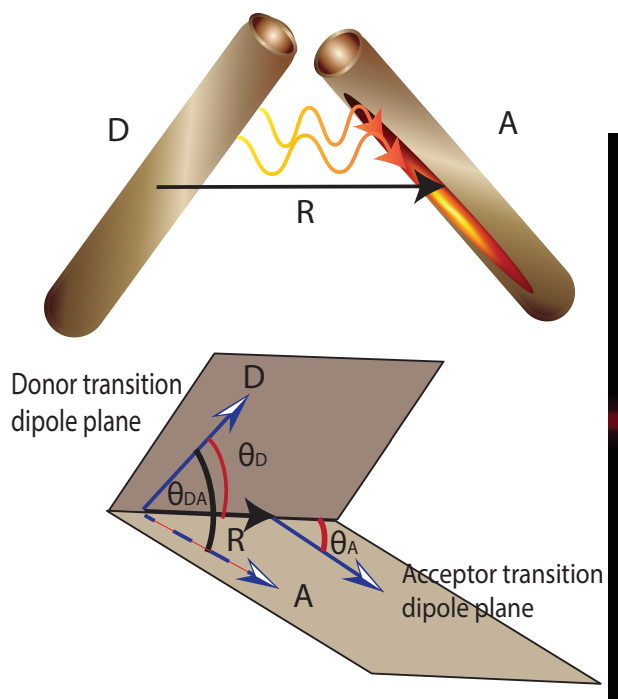

(a)

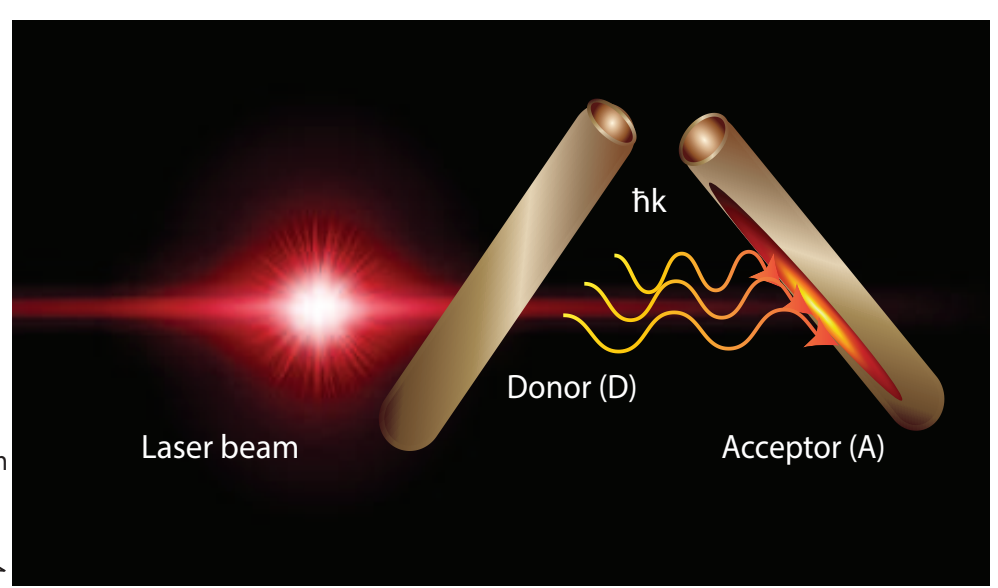

(b)

FIG. 3. Schematics for the (a) direct resonance energy transfer in NW to NW with the orientational factors in Eq. (12), $R$ is the distance between two NWs; (b) laser assisted resonance energy transfer in a NW pair.

tegration and by the residue theorem yields

$$
\begin{gathered}
M_{F I}^{d}=\frac{\mu_{i}^{0 \alpha}(D) \mu_{j}^{\beta 0}(A)}{8 \pi^{2} L \epsilon_{0}}\left(-\nabla^{2} \delta_{i j}+\nabla_{i} \nabla_{j}\right) \\
\int_{0}^{\infty} \int_{0}^{2 \pi} \frac{H_{0}^{(1)}(p R)}{k-p}-\frac{H_{0}^{(2)}(p R)}{k+p} d \phi d p \\
M_{N W}^{(2)}=\frac{\mu_{i}^{0 \alpha}(D) \mu_{j}^{\beta 0}(A)}{4 L \epsilon_{0}}\left[p \delta_{i j}\left\{-Y_{2}(p R)+\frac{Y_{1}(p R)}{p R}\right\}-\right. \\
\left.p \hat{R}_{i} \hat{R}_{j}\left(-Y_{2}(p R)+\frac{Y_{1}(p R)}{p R}\right)\right\}-i p \delta_{i j}\left\{-J_{2}(p R)+\right. \\
\left.\frac{J_{1}(p R)}{p R}\right\}+i p\left\{J_{1}(p R)\left(\frac{\delta_{i j}-\hat{R}_{i} \hat{R}_{j}}{R}\right)+\right. \\
\left.\left.p \hat{R}_{i} \hat{R}_{j}\left(-J_{2}(p R)+\frac{J_{1}(p R)}{p R}\right)\right\}\right]
\end{gathered}
$$

where $Y_{z}(p R), J_{z}(p R)$ are second and first kind of Bessel functions respectively, $z$ is the order number.

We now deploy the asymptotic series for $0<p R \ll$ $\sqrt{ } n+1$ and $n \neq 0[37]$

By applying short-range limits $0<p R \ll 1$ on Eq. (10) yields

$$
\begin{aligned}
M_{N W}^{(2)} & =\frac{\mu_{i}^{0 \alpha}(D) \mu_{j}^{\beta 0}(A)}{2 \pi L \epsilon_{0} R^{2}}\left(\delta_{i j}-2 \hat{R}_{i} \hat{R}_{j}\right) \\
& =\frac{\kappa_{N W}\left|\mu_{i}^{0 \alpha}(D)\right|\left|\mu_{j}^{\beta 0}(A)\right|}{2 \pi L \epsilon_{0} R^{2}}
\end{aligned}
$$

The above equation is dominant in the short-range region and indicates a highly 'virtual' character of the electromagnetic mediator. Here, $\left|\kappa_{N W}\right|^{2}$ is an orientation factor, which describes the influence of the relative orientations of the transition dipole moments of the donor and acceptor NWs, as given by

$$
\begin{aligned}
\kappa_{N W} & =\hat{\mu}_{i}^{0 \alpha}(D)\left(\delta_{i j}-2 \hat{R}_{i} \hat{R}_{j}\right) \hat{\mu}_{j}^{\beta 0}(A) \\
& =\cos \left(\theta_{D A}\right)-2 \cos \left(\theta_{D}\right) \cos \left(\theta_{A}\right)
\end{aligned}
$$

where $\theta_{D}$ is the angle between donor and separation vector $(\boldsymbol{R})$, and $\theta_{A}$ is the angle between acceptor and $\boldsymbol{R}$. $\theta_{D A}$ is the angle between donor and acceptor NWs (see Fig.3). Due to the cylindrical symmetry and the physical nature of the exchanged photon virtue in the $2 \mathrm{D}$ geometry, the orientation factor varies from $0 \leq \kappa_{N W}^{2} \leq 1$ [28].

In a similar manner, the quantum amplitude arising from the input auxiliary beam is given by

$$
\begin{array}{r}
M_{N W}^{(4)}=-\frac{n \hbar c k}{2 \epsilon_{0} V}\left\{e_{i}^{\lambda}(\boldsymbol{k}) \alpha_{i j}^{0 \alpha(D)}(k) \frac{\left(\delta_{j k}-2 \hat{R}_{j} \hat{R}_{k}\right)}{2 \pi L \epsilon_{0} R^{2}}\right. \\
\times \alpha_{k l}^{\beta 0(A)}(k) \bar{e}_{l}^{\lambda}(\boldsymbol{k})+\bar{e}_{i}^{\lambda}(\boldsymbol{k}) \alpha_{i j}^{0 \alpha(D)}(k) \frac{\left(\delta_{j k}-2 \hat{R}_{j} \hat{R}_{k}\right)}{2 \pi L \epsilon_{0} R^{2}} \\
\times \alpha_{k l}^{\beta 0(A)}(k) e_{l}^{\lambda}(\boldsymbol{k})+e_{i}^{\lambda}(\boldsymbol{k}) \bar{e}_{j}^{\lambda}(\boldsymbol{k}) \beta_{i j k}^{0 \alpha(D)}(k) \\
\quad \times \frac{\left(\delta_{k l}-2 \hat{R}_{k} \hat{R}_{l}\right)}{2 \pi L \epsilon_{0} R^{2}} \times \mu_{l}^{0 \beta(A)}+\mu_{i}^{0 \alpha(D)} \\
\left.\times \frac{\left(\delta_{i j}-2 \hat{R}_{i} \hat{R}_{j}\right)}{2 \pi L \epsilon_{0} R^{2}} \times e_{k}^{\lambda}(\boldsymbol{k}) \bar{e}_{l}^{\lambda}(\boldsymbol{k}) \beta_{j k l}^{0 \alpha(D)}(k)\right\}
\end{array}
$$

where $n$ is the number of photons (proportional to laser intensity) in the quantization volume $V, e$ and $\hbar c k$ 
represent the polarization ( $\bar{e}$ denoting its complex conjugation) and energy of the input photon, respectively. Further, $\alpha_{i j}^{f i}(k)$ and $\beta_{i j k}^{f i}(k)$ are generalized and hyperpolarizability defined as $[38,39]$

$$
\begin{aligned}
& \alpha_{i j}^{f i}(k)=\sum_{s}\left\{\frac{\mu_{i}^{f s} \mu_{j}^{s i}}{E_{s f}-\hbar c k}+\frac{\mu_{j}^{f s} \mu_{i}^{s i}}{E_{s i}+\hbar c k}\right\} \\
& \beta_{i j k}^{f i}(k)=\sum_{s, t}\left\{\frac{\mu_{i}^{f t} \mu_{j}^{t s} \mu_{k}^{s i}}{\left(E_{s i}-\hbar c k\right)\left(E_{t i}-\hbar c k\right)}+\right. \\
& \frac{\mu_{i}^{f t} \mu_{k}^{t s} \mu_{j}^{s i}}{\left(E_{s i}-\hbar c k\right)\left(E_{t i}-\hbar c k\right)}+\frac{\mu_{j}^{f t} \mu_{i}^{t s} \mu_{k}^{s i}}{\left(E_{s i}-\hbar c k\right)\left(E_{t i}-\hbar c k\right)}+ \\
& \frac{\mu_{j}^{f t} \mu_{k}^{t s} \mu_{i}^{s i}}{\left(E_{s i}-\hbar c k\right)\left(E_{t i}-\hbar c k\right)}+\frac{\mu_{k}^{f t} \mu_{i}^{t s} \mu_{j}^{s i}}{\left(E_{s i}-\hbar c k\right)\left(E_{t i}-\hbar c k\right)}+ \\
& \left.\frac{\mu_{k}^{f t} \mu_{j}^{t s} \mu_{i}^{s i}}{\left(E_{s i}-\hbar c k\right)\left(E_{t i}-\hbar c k\right)}\right\}
\end{aligned}
$$

The Eq. (13) represents the optically nonlinear influence of the input beam, involves four components: (i) laser photon absorption at $D$, stimulated emission at $A$; (ii) the converse; (iii) absorption and emission at $D$; and (iv) both photon events at $A$.

According to Fermi's Golden Rule given in Eq. (8), the matrix elements of the interaction operator between the initial and final states of the field determine the transition probability

$$
\begin{aligned}
\Gamma_{N W}^{\text {laser }}=\frac{2 \pi \rho}{\hbar} & \left|M_{N W}^{(2)}\right|^{2}+\frac{2 \pi \rho}{\hbar}\left|M_{N W}^{(4)}\right|^{2} \\
& +\frac{4 \pi \rho}{\hbar} \operatorname{Re}\left\{M_{N W}^{(2)} \bar{M}_{N W}^{(4)}\right\}
\end{aligned}
$$

The first term represents the normal Förster energy transfer rate

$$
\Gamma_{N W}^{(2-2)}=\frac{\left|\mu_{i}^{0 \alpha}(D)\right|^{2}\left|\mu_{j}^{\beta 0}(A)\right|^{2}\left|\kappa_{N W}\right|^{2} \rho}{2 \pi L^{2} R^{4} \epsilon_{0}^{2} \hbar}
$$

The middle term depends on the laser intensity, and explicitly given by

$$
\begin{array}{r}
\Gamma_{N W}^{(4-4)}=\frac{I^{2}(k) \rho}{8 L^{2} \hbar c^{2} \pi \epsilon_{0}^{4} R^{4}} \mid e_{i}^{\lambda}(\boldsymbol{k}) \alpha_{i j}^{0 \alpha(D)}(k) \\
\times\left(\delta_{j k}-2 \hat{R}_{j} \hat{R}_{k}\right) \alpha_{k l}^{\beta 0(A)}(k) \bar{e}_{l}^{\lambda}(\boldsymbol{k})+\bar{e}_{i}^{\lambda}(\boldsymbol{k}) \alpha_{i j}^{0 \alpha(D)}(k) \\
\times\left(\delta_{j k}-2 \hat{R}_{j} \hat{R}_{k}\right) \alpha_{k l}^{\beta 0(A)}(k) e_{l}^{\lambda}(\boldsymbol{k})+e_{i}^{\lambda}(\boldsymbol{k}) \bar{e}_{j}^{\lambda}(\boldsymbol{k}) \beta_{i j k}^{0 \alpha(D)}(k) \\
\times\left(\delta_{k l}-2 \hat{R}_{k} \hat{R}_{l}\right) \mu_{l}^{0 \beta(A)}+\mu_{i}^{0 \alpha(D)}\left(\delta_{i j}-2 \hat{R}_{i} \hat{R}_{j}\right) \\
\times\left.\beta_{j k l}^{0 \alpha(D)}(k) e_{k}^{\lambda}(\boldsymbol{k}) \bar{e}_{l}^{\lambda}(\boldsymbol{k})\right|^{2}
\end{array}
$$

$I(k) \equiv n \hbar c^{2} k / V$ is the auxiliary laser intensity, described as comprising $n$ photons with wave-vector $\boldsymbol{k}$. The third term, linear in $I$, signifies a quantum interference of these two concurrent processes

$$
\begin{array}{r}
\Gamma_{N W}^{i n t e r}=-\frac{I(k)\left|\mu_{i}^{0 \alpha}(D) \| \mu_{j}^{\beta 0}(A)\right| \kappa_{N W} \rho}{2 L^{2} \hbar c \pi \epsilon_{0}^{3} R^{4}} \\
\times R e\left\{e_{i}^{\lambda}(\boldsymbol{k}) \alpha_{i j}^{0 \alpha(D)}(k)\left(\delta_{j k}-2 \hat{R}_{j} \hat{R}_{k}\right) \alpha_{k l}^{\beta 0(A)}(k) \bar{e}_{l}^{\lambda}(\boldsymbol{k})\right. \\
+\bar{e}_{i}^{\lambda}(\boldsymbol{k}) \alpha_{i j}^{0 \alpha(D)}(k)\left(\delta_{j k}-2 \hat{R}_{j} \hat{R}_{k}\right) \alpha_{k l}^{\beta 0(A)}(k) e_{l}^{\lambda}(\boldsymbol{k}) \\
+e_{i}^{\lambda}(\boldsymbol{k}) \bar{e}_{j}^{\lambda}(\boldsymbol{k}) \beta_{i j k}^{0 \alpha(D)}(k)\left(\delta_{k l}-2 \hat{R}_{k} \hat{R}_{l}\right) \mu_{l}^{0 \beta(A)} \\
\left.+\mu_{i}^{0 \alpha(D)}\left(\delta_{i j}-2 \hat{R}_{i} \hat{R}_{j}\right) \beta_{j k l}^{0 \alpha(D)}(k) e_{k}^{\lambda}(\boldsymbol{k}) \bar{e}_{l}^{\lambda}(\boldsymbol{k})\right\}
\end{array}
$$

By implementing conservative estimates for parameters, $\mu^{0 \alpha}(D) \approx \mu^{\beta 0}(A) \approx 5 \times 10^{-30} \mathrm{C} \mathrm{m}$ for transition dipole moment magnitudes and $\alpha(D) \alpha(A) \approx$ $\beta(D) \mu(A) \approx \mu(D) \beta(A) \approx 1 \times 10^{-78} \mathrm{C}^{4} \mathrm{~m}^{2} \mathrm{~J}^{-2}$ for tensor products, we can estimate the relative magnitude of the effect with increasing laser intensity. We use above parameters for the development of the plots in Sec. III A, III B.

\section{Laser irradiance and orientational dependence on transfer rate}

We envisage three vital orientational factors and the laser irradiance dependence on each case to enhance the transfer rate.

1. $\kappa_{N W}=1$ : When $\cos \left(\theta_{D A}\right)=\pi, \cos \left(\theta_{D}\right)=\pi$ (or 0 ) and $\cos \left(\theta_{A}\right)=0$ (or $\pi$ ), orientation factor $\kappa_{N W}$ becomes 1. Fig. 5 (a) illustrates the total energy transfer rate and the magnitudes of the individual contributions to the transfer rate with increasing laser intensity. The direct RET rate is constant with respect to $I$ as non-mediated RET is independent of the radiation intensity. The LARET gradually increases with $I$, showing the quadratic nature of $\Gamma_{N W}^{4-4}$. Nevertheless, quantum interference $\left(\Gamma_{N W}^{i n t e r}\right)$, leanerly decreases with $I$, owing to the quantum amplitudes of direct and laser mediated RET (see Fig. 4 (a) and Eq. (19)). These results exhibit a gradual decline of the total energy transfer efficiency until it reaches its minimum point when $I$ becomes $1.33 \times 10^{17} \mathrm{Wm}^{-2}$. After this point, the total energy transfer rate steadily elevates its value and finally starts dominating the direct transfer rate when $I \geqslant 2.66 \times 10^{17} \mathrm{Wm}^{-2}$ (see point $X$ in Fig. 5 (a)).

2. $\kappa_{N W}=0$ : This is when both transition dipole moments are perpendicular to each other and perpendicular to the donor-acceptor separation vector. This configuration prohibits the direct transfer of energy, hence it excludes the quantum interference. Therefore, only laser driven RET term survives, exhibiting a steady increment of total energy transfer 
rate with respect to $I$. Transfer rates and corresponding quantum amplitudes are depicted in Fig. 5 (b) and Fig. 4 (b) respectively.

3. $\boldsymbol{\kappa}_{\boldsymbol{N} \boldsymbol{W}}=\mathbf{- 1}$ : When $\cos \left(\theta_{D A}\right)=\cos \left(\theta_{D}\right)=$ $\cos \left(\theta_{A}\right)=0$, orientation factor $\kappa_{N W}$ becomes -1 . In this case, the coupling matrix element of direct RET acquires a negative value as illustrated in Fig. 4 (c), delivering a positive quantum interference. Analogous to the case of $\kappa_{N W}=1$, laser mediated RET gradually increases as a function of $I$ and direct RET is independent of $I$. Thus, the total rate enhances steadily with increasing $I$, displaying a higher total transfer rate compared to the Förster type direct RET for the whole spectrum of laser intensities. These results are illustrated in Fig. 5 (c).

\section{B. Quantum dot System}

In contrast to molecular fluorophores, individual QDs have discrete atom-like discrete energy transitions, which arise from band splitting due to the quantum confinement effect. In particular, owing to its unique optical properties, QDs are being exploited as highly efficient fluorophores, typically possessing excellent quantum yields and photostability. Furthermore, QDs exhibit excellent photophysical properties that are highly desired in a RET system, including: (1) broad absorption spectra; (2) large absorption cross-sections; (3) narrow, size-tunable emission spectra to name a few [40-42]. In this section, a pair of QDs separated by distance $R$ interacting with a laser field is considered. The direct interaction between the donor and acceptor can be calculated from the second order quantum amplitude [27, 43]

$$
\begin{array}{r}
M_{Q D}^{(2)}=\frac{\mu_{i}^{0 \alpha}(D) \mu_{j}^{\beta 0}(A)}{16 \pi^{3} \epsilon_{0}}\left(-\nabla^{2} \delta_{i j}+\nabla_{i} \nabla_{j}\right) \int_{0}^{\infty} \int_{0}^{2 \pi} \int_{-1}^{1} \\
\times\left\{\frac{e^{i \boldsymbol{p} \cdot \boldsymbol{R}} p}{k-p}-\frac{e^{-i \boldsymbol{p} \cdot \boldsymbol{R}} p}{k+p}\right\} d(\cos \theta) d \phi d p
\end{array}
$$

Performing contour integration with a suitable contour and by the residue theorem yields

$$
M_{Q D}^{(2)}=\mu_{i}^{0 \alpha}(D) \frac{\delta_{i j}-3 \hat{R}_{i} \hat{R}_{j}}{4 \pi \epsilon_{0} R^{3}} \mu_{j}^{\beta 0}(A)
$$

The application of the Fermis golden rule gives rise to the following expression for the direct energy transfer rate between two QDs:

$$
\Gamma_{Q D}^{(2-2)}=\frac{\left|\mu_{i}^{0 \alpha}(D)\right|^{2}\left|\mu_{j}^{\beta 0}(A)\right|^{2}\left|\kappa_{Q D}\right|^{2} \rho}{8 \pi R^{6} \epsilon_{0}{ }^{2} \hbar}
$$

where $\left|\kappa_{Q D}\right|^{2}$ is an orientation factor

$$
\begin{aligned}
\kappa_{Q D} & =\hat{\mu}_{i}^{0 \alpha}(D)\left(\delta_{i j}-3 \hat{R}_{i} \hat{R}_{j}\right) \hat{\mu}_{j}^{\beta 0}(A) \\
& =\cos \left(\theta_{D A}\right)-3 \cos \left(\theta_{D}\right) \cos \left(\theta_{A}\right)
\end{aligned}
$$

where $\theta_{D}$ is the angle between donor and separation vector $(\boldsymbol{R})$, and $\theta_{A}$ is the angle between acceptor and $\boldsymbol{R}$. $\theta_{D A}$ is the angle between donor and acceptor QDs. Owing to the spherical symmetry of the QDs, the orientation factor varies from $0 \leq \kappa_{Q D}^{2} \leq 4$.

The indirect transfer rate $\Gamma_{Q D}^{(4-4)}$, represents the optically nonlinear influence of the input beam, is delivered by previous work [30], [44]

$$
\begin{array}{r}
\Gamma_{Q D}^{(4-4)}=\frac{I^{2}(k) \rho}{32 \hbar c^{2} \pi \epsilon_{0}^{4} R^{6}} \mid e_{i}^{\lambda}(\boldsymbol{k}) \alpha_{i j}^{0 \alpha(D)}(k) \\
\times\left(\delta_{j k}-3 \hat{R}_{j} \hat{R}_{k}\right) \alpha_{k l}^{\beta 0(A)}(k) \bar{e}_{l}^{\lambda}(\boldsymbol{k})+\bar{e}_{i}^{\lambda}(\boldsymbol{k}) \alpha_{i j}^{0 \alpha(D)}(k) \\
\times\left(\delta_{j k}-3 \hat{R}_{j} \hat{R}_{k}\right) \alpha_{k l}^{\beta 0(A)}(k) e_{l}^{\lambda}(\boldsymbol{k})+e_{i}^{\lambda}(\boldsymbol{k}) \bar{e}_{j}^{\lambda}(\boldsymbol{k}) \beta_{i j k}^{0 \alpha(D)}(k) \\
\times\left(\delta_{k l}-3 \hat{R}_{k} \hat{R}_{l}\right) \mu_{l}^{0 \beta(A)}+\mu_{i}^{0 \alpha(D)}\left(\delta_{i j}-3 \hat{R}_{i} \hat{R}_{j}\right) \\
\left.\beta_{j k l}^{0 \alpha(D)}(k)\right|^{2} \times e_{k}^{\lambda}(\boldsymbol{k}) \bar{e}_{l}^{\lambda}(\boldsymbol{k})
\end{array}
$$

Thus, the quantum interference due to the laser field is given by

$$
\begin{array}{r}
\Gamma_{Q D}^{i n t e r}=-\frac{I(k)\left|\mu_{i}^{0 \alpha}(D) \| \mu_{j}^{\beta 0}(A)\right| \kappa_{Q D} \rho}{8 \hbar c \pi \epsilon_{0}^{3} R^{6}} \\
\times R e\left\{e_{i}^{\lambda}(\boldsymbol{k}) \alpha_{i j}^{0 \alpha(D)}(k)\left(\delta_{j k}-3 \hat{R}_{j} \hat{R}_{k}\right) \alpha_{k l}^{\beta 0(A)}(k) \bar{e}_{l}^{\lambda}(\boldsymbol{k})\right. \\
+\bar{e}_{i}^{\lambda}(\boldsymbol{k}) \alpha_{i j}^{0 \alpha(D)}(k)\left(\delta_{j k}-3 \hat{R}_{j} \hat{R}_{k}\right) \alpha_{k l}^{\beta 0(A)}(k) e_{l}^{\lambda}(\boldsymbol{k}) \\
+e_{i}^{\lambda}(\boldsymbol{k}) \bar{e}_{j}^{\lambda}(\boldsymbol{k}) \beta_{i j k}^{0 \alpha(D)}(k)\left(\delta_{k l}-3 \hat{R}_{k} \hat{R}_{l}\right) \mu_{l}^{0 \beta(A)} \\
\left.+\mu_{i}^{0 \alpha(D)}\left(\delta_{i j}-3 \hat{R}_{i} \hat{R}_{j}\right) \beta_{j k l}^{0 \alpha(D)}(k) e_{k}^{\lambda}(\boldsymbol{k}) \bar{e}_{l}^{\lambda}(\boldsymbol{k})\right\}
\end{array}
$$

1. Laser irradiance and orientational dependence on RET rate

Similar to the NW systems, we study three non-trivial orientational factors and the laser irradiance dependence on each case to enhance the transfer rate.

1. $\kappa_{Q D}=2$ : Similar to the NW case, laser modified coupling matrix element $\left(M_{Q D}^{(4-4)}\right)$ decreases with intensity while direct coupling remains static for all the laser intensities, producing a negative quantum interference value which steadily declines as a function of $I$. This is shown in Fig. 7 (a). As depicted in Fig. 8 (a), the LARET in QDs gradually increases with $I$. On the otherhand quantum interference experienced a steady drop when $I$ increases, displaying a gradual decline in the total energy transfer rate until $I$ reaches its value of $2.66 \times 10^{17} \mathrm{Wm}^{-2}$. It is immediately apparent 


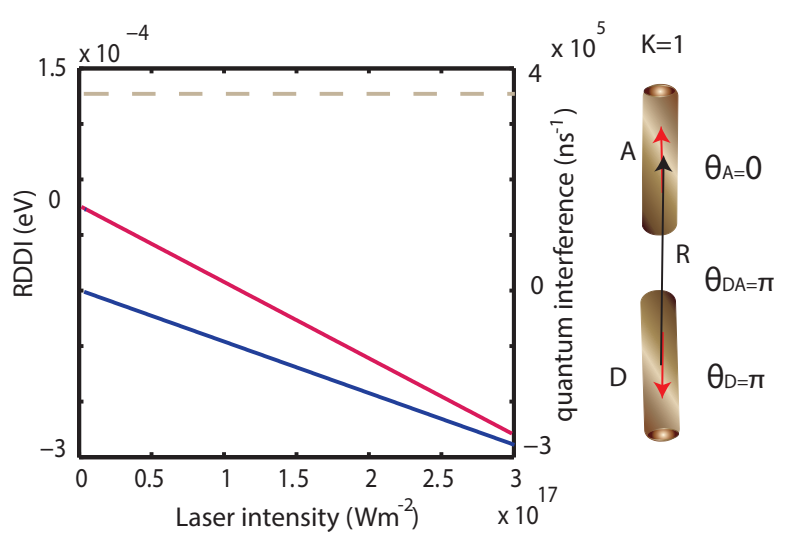

(a)

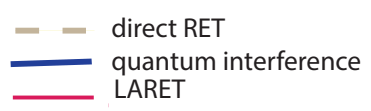

FIG. 4. RDD engths of the direct RET (ash colour line), laser driven RET (pink colour line) and the quantum interference (blue colour line) when $\kappa=1,0,-1$ are shown in (a)-(c) respectively as a function of the laser intensity

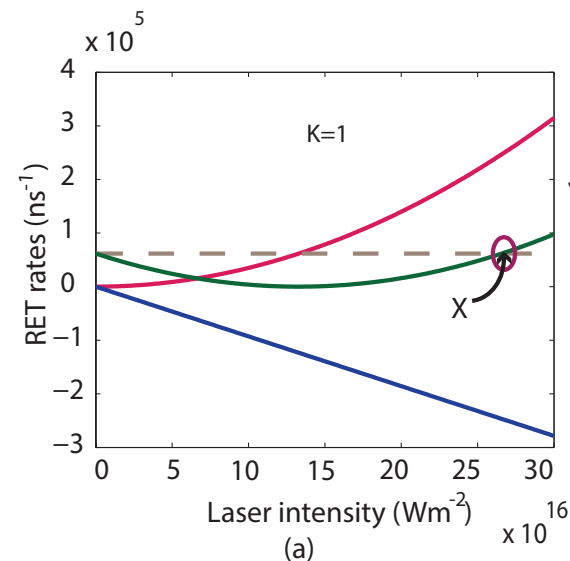

(a)

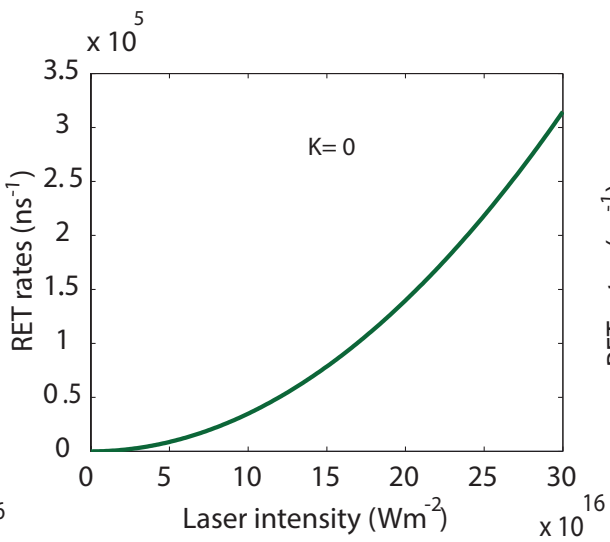

(b)

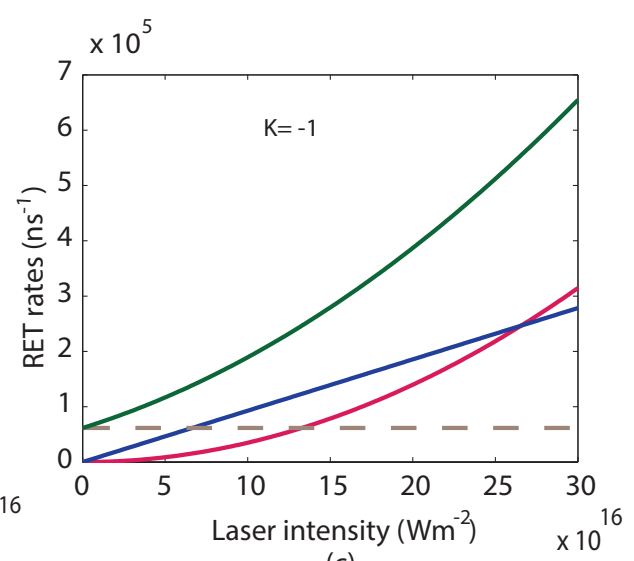

(c)

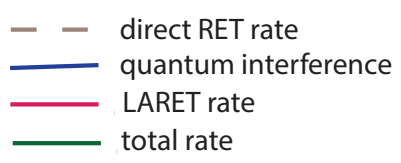

FIG. 5. Transfer rates of the direct RET (ash colour line), laser driven RET (red colour line), quantum interference (blue colour line) and the total LARET rate (green colour line) when $\kappa=1,0,-1$ are shown in (a)-(c) respectively as a function of the laser intensity 

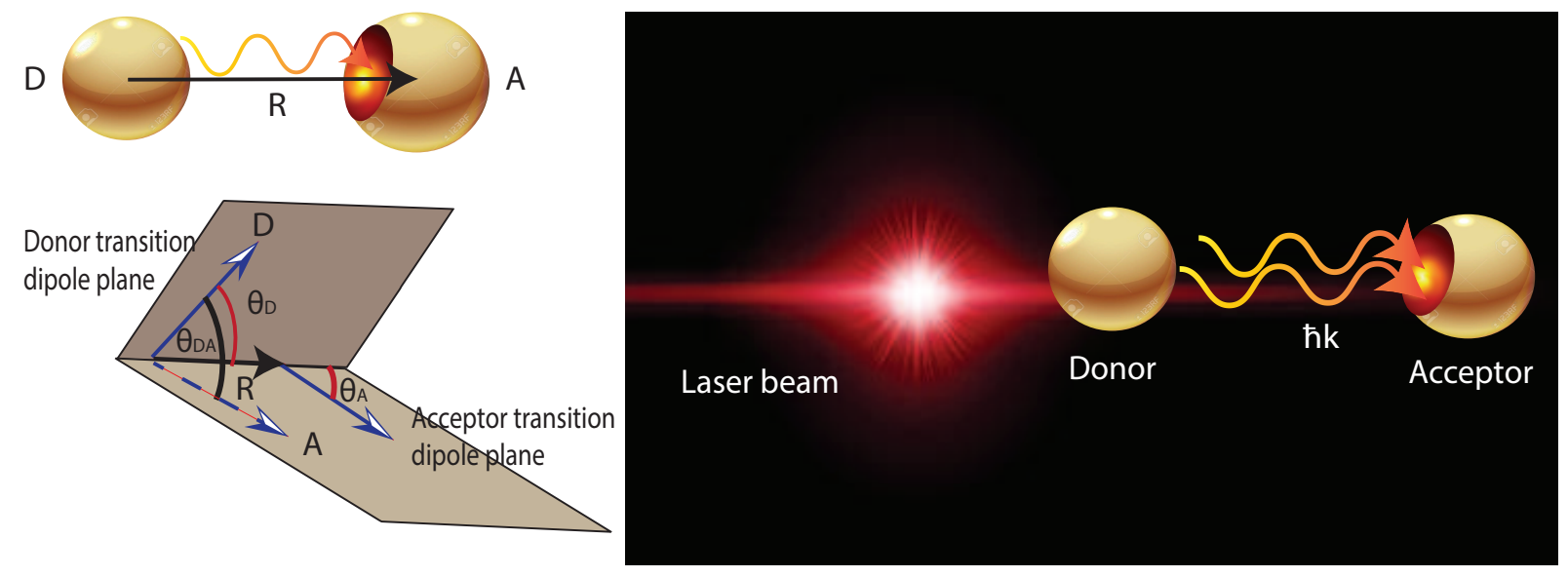

(a)

(b)

FIG. 6. Schematics for the (a) direct resonance energy transfer in QD to QD with the orientational factors in Eq. (23), $R$ is the distance between two QDs; (b) laser assisted resonance energy transfer in a QD pair.

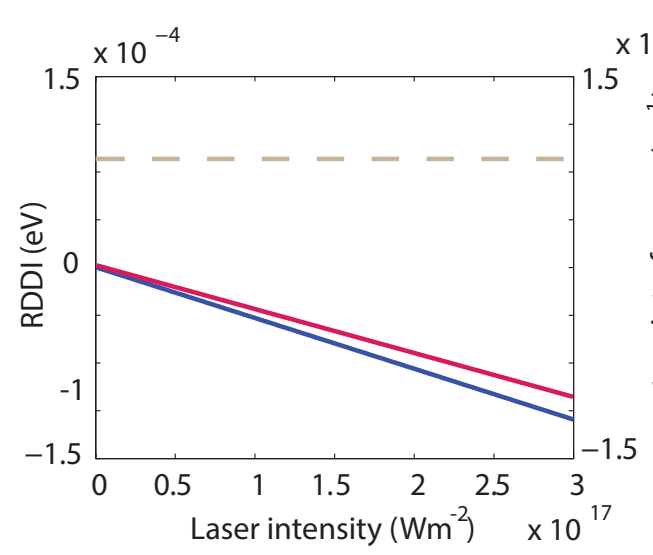

(a)

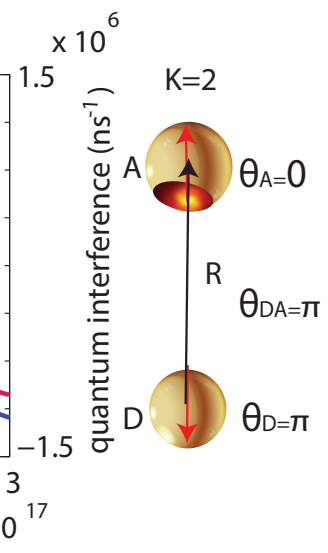

$0 \times 10^{-4}$

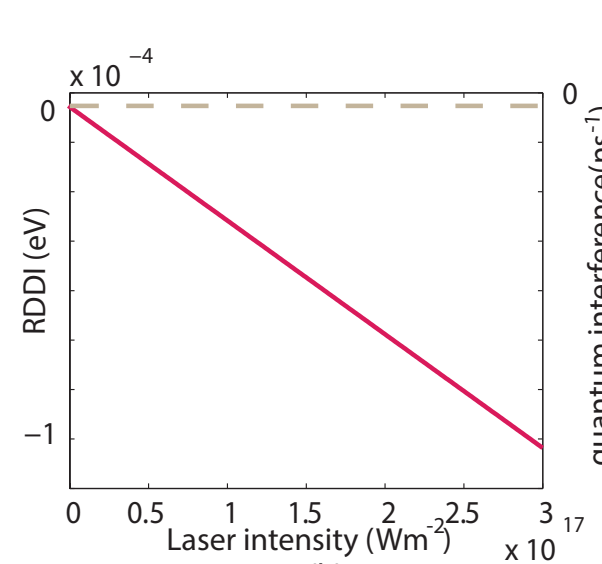

(b)
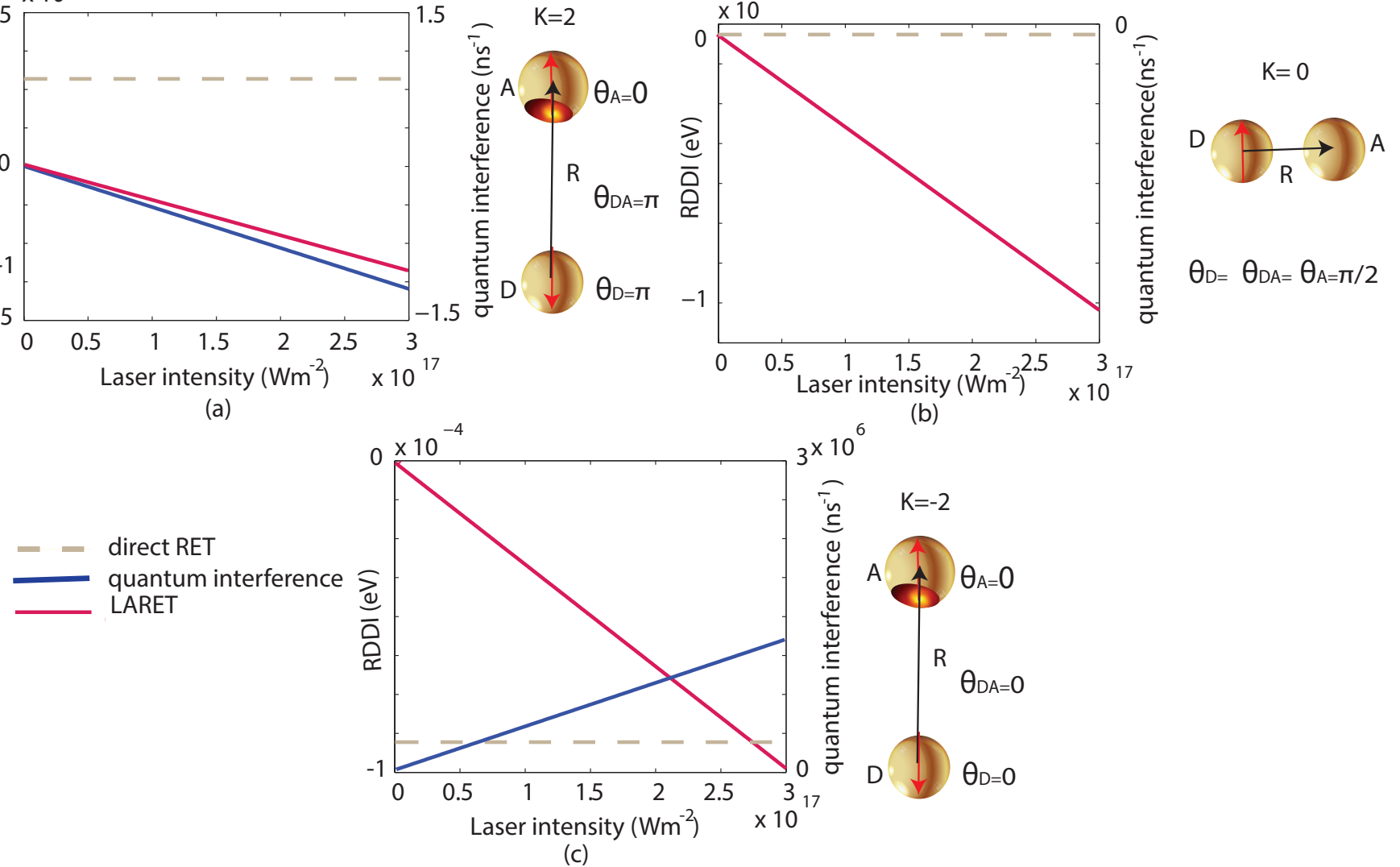

FIG. 7. RDDI strengths of the direct RET (ash colour line), laser driven RET (pink colour line) and the quantum interferenc $\bigcirc$ (blue colour line) when $\kappa=2,0,-2$ are shown in (a)-(c) respectively as a function of the laser intensity

that, at intensities less than $2.66 \times 10^{17}$ v 2 , the transfer is Förster dominated. Due to the effect of LARET, total energy transfer elevates with increasing $I$ and finally achieves 1 her transfer efficiencies when $I \geqslant 5.32 \times 10^{17} \vee{ }^{2}$ (see point $Y$ in Fig. $8(\mathrm{a}))$

2. $\kappa_{Q D}=\mathbf{0}$ : This case is analogous to the case of $\kappa_{N W}=0$. Here the direct coupling has been "switched off" between QD particles by arranging 


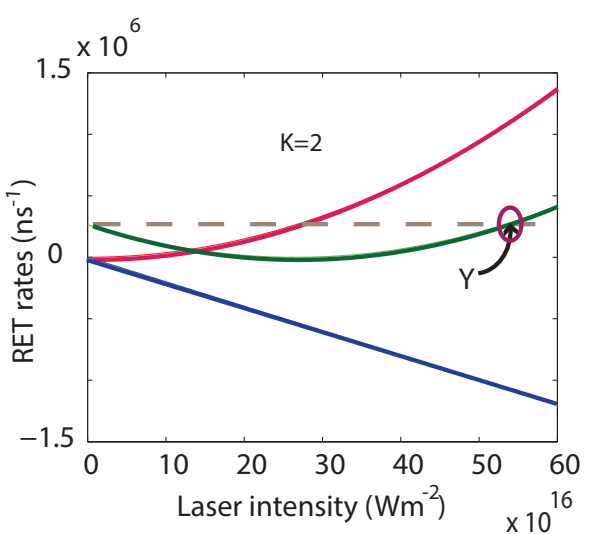

(a)

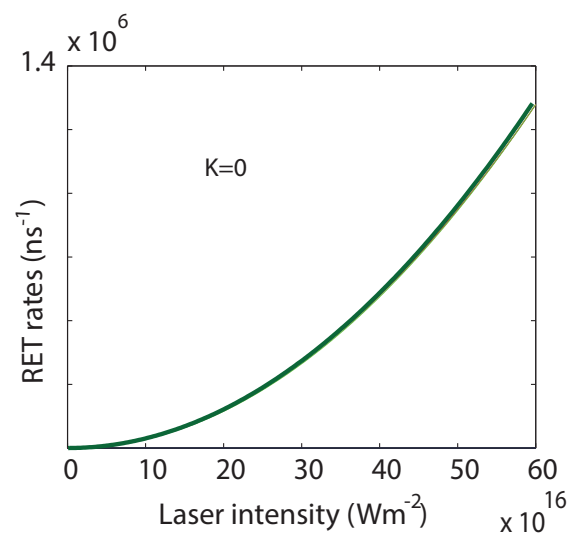

(b)

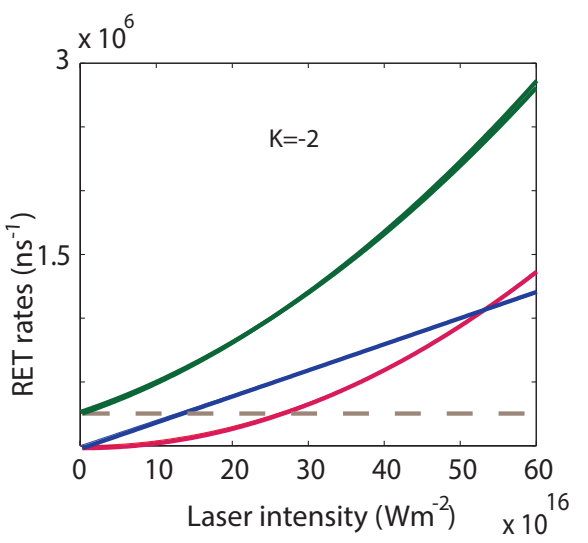

(c)

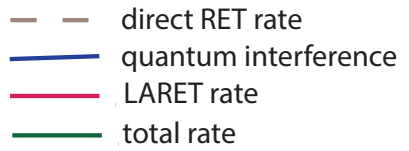

FIG. 8. Transfer rates of the direct RET (ash colour line), laser driven RET (red colour line), quantum interference (blue colour line) and the tgtal LARET rate (green colour line) when $\kappa=2,0,-2$ are shown in (a)-(c) respectively as a function of the laser intensity $\Omega$

them such that the transition dipole moments are perpendicular to each other and donor-acceptor separation vector, hence excluding the quantum interference. Therefore, the total energy transfer only contains the contribution of laser dependent RET, which gradually increases as a function of $I$. These results are shown in Fig. 7 (b), Fig. 8 (b).

3. $\kappa_{Q D}=\mathbf{- 2}$ : This is when both transition dipole moments are parallel to each other and parallel to the donor-acceptor separation vector. The quantum amplitude of direct RET acquires a negative value as depicted in Fig. 7 (c), resulting in a positive quantum interference. Analogous to the case of $\kappa_{Q D}=2$, laser mediated RET gradually increases as a function of $I$ and direct RET is independent of $I$. Therefore, the total rate enhances steadily with $I$. Here, the direct RET between a pair of QDs is dominated by the LARET for the whole spectrum of laser intensities. These results are shown in Fig. 8 (c). However, unfocused light does not perturb RET to a significant exten $\Omega$

\section{Design guidelines for high efficiency laser driven RET}

The RET rate can be enhanced efficiently, when the optimal configuration of the nanostructure system is implemented. Here, for fast transfer between nearby sites are those where the particle transition dipole moments and the separation vector are collinear $\left(\theta_{D A}=\theta_{D}=\right.$
$\left.\theta_{A}=0\right)$. The transfer rate enhancement with increasing laser intensity for this scenario is depicted in Fig. 9 (a), comparing both QD and NW cases. Then werformed calculation to perceive RET rate enhancement as a percentage (Fig. $9(\mathrm{~b}))$. The results demonstrate that even for moderate laser intensities $\left(I \approx 10^{16}\right)$, up to $70 \%$ and $30 \%$ rate enhancements can be achieved for QDs and NWs respectively.

Merestigated the distance dependence in laser driven RET in NWs and QDs. Owing to the $(R)^{-4}$ and $(R)^{-6}$ distance dependence on the total transfer rate in NW and QD respectively, lower relative spacing between donor and acceptor particles displays higher transfer efficiency. This is shown in Fig. 9 (c). Further, it can also be observed from the plots, that QDs produce higher LARET efficiency in relatively lower intensities. However, when increasing the laser beam intensity, LARET in NWs starts to outperform QDs.

\section{DISCUSSION}

In Sec. (III), laser driven resonance energy transfer rate equations, corresponding quantum amplitudes and quantum interferences for nanowire and quantum dot systems have been derived and the results are diseussed with figures. The results have demonstrated that the transfer of energy can be enhanced to a significant and measurable degree when the laser intensity is pulsed and highly focused or through deploying favourable configurations of the nanostructure particles $\left(\kappa_{N W}=-1, \kappa_{Q D}=-2\right)$. It is also important that the 


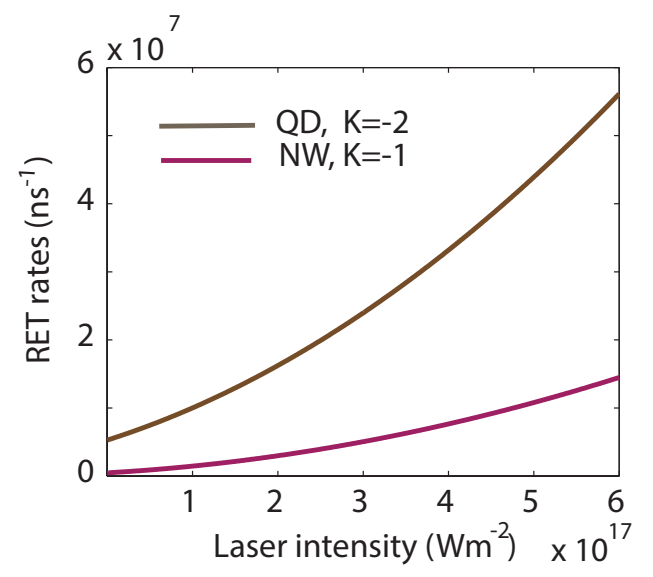

(a)

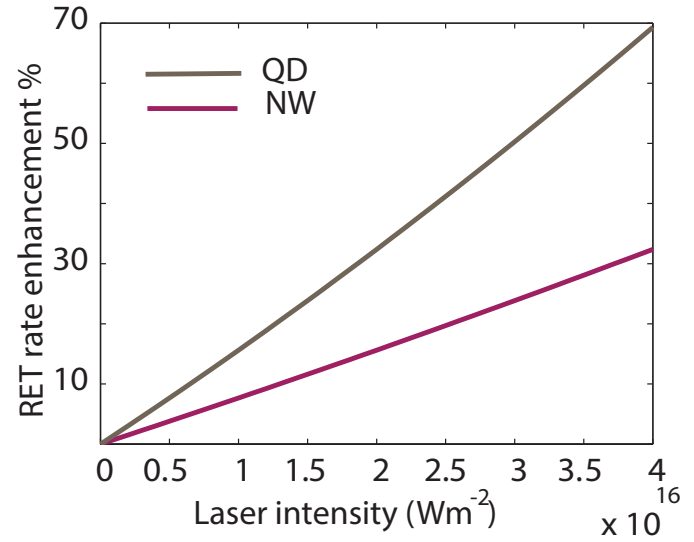

(b)

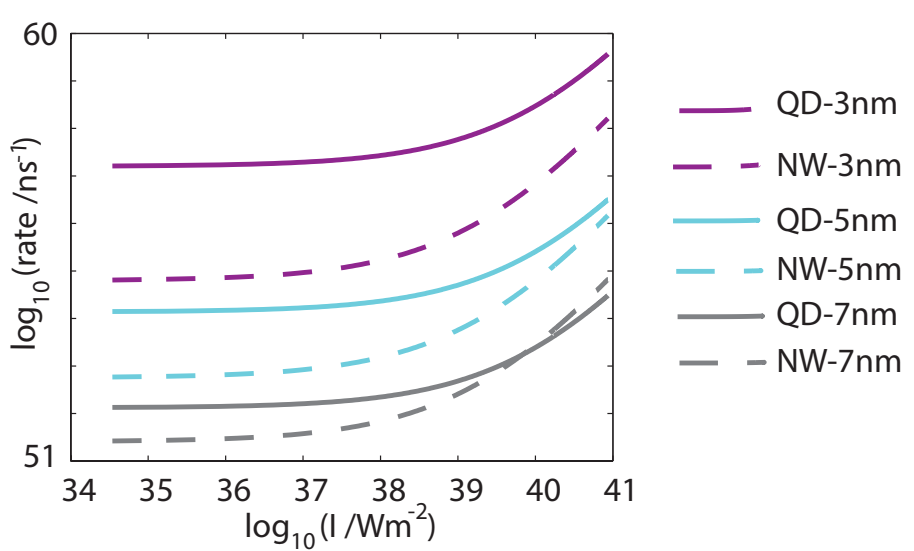

(c)

FIG. 9. (a) The brown colour and purple colour-solid lines represent the LARET rates when $\kappa_{N W}=-1$ and $\kappa_{Q D}=-2$; (b) RET rate enhancement factor in QD and NW systems; (c) Log-Log plot of transfer rate as a function of log $(I)$, for three different relative distances $(3 \mathrm{~nm}, 5 \mathrm{~nm}, 7 \mathrm{~nm})$ between $\mathrm{D}$ and $\mathrm{A}$, dash lines represent the transfer rates for the case of NW pair.

laser radiation is off-resonant, in order to prevent direct excitation of the acceptor.

Exploration of the quantum amplitudes in laser assistant RET is the core contribution of all derivations. Our calculations for quantum amplitude are based on Schrödinger state vector representation of quantum dynamics, where matrix element for RET is represented as a sum of differently time-ordered contributions [23]. Fig. 4(a)-(c) indicate the variation of RDDI strengths in a NW pair against the laser irradiance for different orientation factors. Interestingly, laser driven RDDIs are identical for all three cases. This is because quantum amplitude is independent of the orientational factor. Nevertheless, direct RET and quantum interference vary with $\kappa$, and vanish when NWs are perpendicular to each other and to the distance vector. The variations of RDDIs of QDs with laser irradiation and orientational factor exhibit similar patterns observed for NWs (Figs. 7 (a)-(c)), with one difference. Due to the physical nature and geometry of the quantum dots, the $\kappa_{Q D}$ value is higher than the $\kappa_{N W}$.
Furthermore, rates for unmediated RET, laser driven RET and the quantum interference for a pair of NWs are illustrated in Fig. 5 (a)-(c) for three non trivial orientational factors. In Fig. 5 (a), where the $\Gamma^{t o t}$ is plotted against laser irradiation when $\kappa_{N W}=1$, a gradual decrement and then an increment of total transfer rate with the laser intensity can be observed. Here, when the laser intensity is low, quantum interference dominates the transfer rate. However, when the intensity increases, laser assisted RET dominates the transfer process and finally for high intensities, an enhancement in RET rate can be observed. Moreover, when $\kappa_{N W}=0$ (Fig. 5 (b)), direct RET and hence the quantum interference are explicitly forbidden, allowing full control to the laser driven transfer. In this case, optical switching can be produced by the throughput of a single off-resonant beam (or, with more control options, by two coincident beams) [44-46]. Further, for angles $\theta_{D A}=\theta_{D}=\theta_{A}=0$, a gradual increase of the total transfer rate against laser intensity can be observed as depicted in Fig. 5 (c). Thus the RET 
rate can be enhanced to a greater extent under this condition. Additionally, the results for QDs show similar patterns (Fig. 8 (a)-(c)) and hold same explanations, with one significant difference. Due to the nature of the real and virtual photon virtue in spherical geometry, when $\kappa_{Q D}=2\left(\theta_{D A}=\theta_{D}=\pi, \theta_{A}=0\right)$, higher laser intensities are needed to suppress the quantum interference which arises from the applied laser beam.

Moreover, by suitably configuring an arrangement of transition dipoles, it proved possible to design optical switches [44, 47]. Here, the optical switching actions can be implemented by exploiting RET mechanism activated by throughput laser radiation of an appropriate intensity, frequency and polarization. Therefore, implemented in a configuration with nanowires (or quantum dots) arrayed in thin films, the process may offer design of logic gates, optical transistor and ultrafast parallelprocessing capabilities etc. This represents scope for potential development of the theory.

\section{CONCLUSIONS AND OUTLOOK}

We calculated the rates of laser driven resonance energy transfer in optically excited systems composed of NWs and QDs. A full quantum electrodynamical treatment for laser assisted energy migration is developed and formulated with the aid of Feynman diagram methods [48]. In a nutshell, The results expounded here indicate that at relatively higher laser intensities, the higher-order effects are significant even in a non-favourable configura- tion of the particles (when donor-acceptor particles make an angle of $\pi$ with each other and one particle is parallel to the donor-acceptor displacement vector). Besides, the results show that, at reasonable levels of laser intensity, quantum interference arises from the auxiliary laser beam drops to insignificant levels, enhancing the total RET rate between nanostuctures. Thus, at suitably higher laser intensities, for example those readily available from a focused, laser or a spaser, energy-transfer rate explicitly enhanced up to $70 \%$.

In addition to the calculations presented in this article, it is viable to envisage high-intensity nanolasers based on spasers to enhance and control the process of RET. In this case, the emitted photons from and into the laser beam are replaced by surface plasmons. The electric field operator for the surface plasmons would modify the quantum amplitudes and the transfer rate, provide a high efficiency controlling mechanism for resonance energy transfer.

The ensuing results open up a new avenue for applications of high-efficiency artificial nano-antenna systems. Furthermore, the proposed laser driven RET mechanism holds potential for optical transistors, optical logic gates, providing building blocks for more complex nanophotonic circuitry.

\section{ACKNOWLEDGMENTS}

The work of D.W. is supported by the Monash University Institute of Graduate Research. The work of M.P. is supported by the Australian Research Council, through its Discovery Grant No. DP140100883.
[1] L. Hu and G. Chen, Nano letters 7, 3249 (2007).

[2] G. Rosaz, B. Salem, N. Pauc, P. Gentile, A. Potié, A. Solanki, and T. Baron, Semiconductor Science and Technology 26, 085020 (2011).

[3] Y.-L. Lee, B.-M. Huang, and H.-T. Chien, Chemistry of Materials 20, 6903 (2008).

[4] B. A. Kairdolf, A. M. Smith, T. H. Stokes, M. D. Wang, A. N. Young, and S. Nie, Annual review of analytical chemistry (Palo Alto, Calif.) 6, 143 (2013).

[5] C. S. Kumarasinghe, M. Premaratne, S. D. Gunapala, and G. P. Agrawal, Scientific reports 6 (2016).

[6] D. Sikdar, I. D. Rukhlenko, W. Cheng, and M. Premaratne, Plasmonics 9, 659 (2014).

[7] M. Premaratne and G. P. Agrawal, Light Propagation in Gain Media (Cambridge University Press, 2011).

[8] S. Brichkin, High Energy Chemistry 47, 277 (2013).

[9] P. L. Hernández-Martínez and A. O. Govorov, Phys. Rev. B 78, $035314(2008)$

[10] V. I. Novoderezhkin, A. Palacios, H. Van Amerongen, and R. Van Grondelle, The Journal of Physical Chemistry B 108, 10363 (2004).

[11] K. Becker, J. M. Lupton, J. Müller, A. L. Rogach, D. V. Talapin, H. Weller, and J. Feldmann, Nature materials 5, 777 (2006).

[12] V. Raicu, Journal of biological physics 33, 109 (2007).
[13] I. Medintz and N. Hildebrandt, FRET-Förster Resonance Energy Transfer: From Theory to Applications (John Wiley \& Sons, 2013).

[14] C. Rupasinghe, I. D. Rukhlenko, and M. Premaratne, ACS nano 8, 2431 (2014).

[15] C. Jayasekara, M. Premaratne, M. I. Stockman, and S. D. Gunapala, J. App. Phy. 118, 173101 (2015).

[16] R. J. Cogdell, A. T. Gardiner, A. W. Roszak, C. J. Law, J. Southall, and N. W. Isaacs, Photosynthesis Research 81, 207 (2004).

[17] M. Mohseni, P. Rebentrost, S. Lloyd, and A. AspuruGuzik, The Journal of ehemical physics 129, 174106 (2008).

[18] S. Zhang, Z. Lü, Y. Peng, Y. Liu, and Y. Yang, Journal of Luminescence 128, 1523 (2008).

[19] G. D. Scholes, G. R. Fleming, A. Olaya-Castro, and R. van Grondelle, Nature ehemistry 3, 763 (2011).

[20] T. Förster, Annalen der Physik 437, 55 (1948).

[21] J. Avery, Proceedings of the Physical Society 88, 1 (1966).

[22] L. Gomberoff and E. A. Power, Proceedings of the Physical Society 88, 281 (1966).

[23] D. P. Craig and T. Thirunamachandran, Molecular Quantum Electrodynamics (Dover, New York, 1998).

[24] A. Salam, Molecular quantum electrodynamics: long- 
range intermolecular interactions (Wiley, Hoboken, NJ, 2010).

[25] J. J. Rodriguez and A. Salam, Chemical Physics Letters 498, 67 (2010).

[26] A. Salam, J. Chem. Phys. 136, 014509 (2012).

[27] D. Weeraddana, M. Premaratne, and D. L. Andrews, Physical Review B 92, 035128 (2015).

[28] D. Weeraddana, M. Premaratne, and D. L. Andrews, Physical Review B 93, 075151 (2016).

[29] D. L. Andrews and J. M. Leeder, The Journal of ehemical physics 130, 034504 (2009).

[30] P. Allcock, R. D. Jenkins, and D. L. Andrews, Physical Review A 61, 023812 (2000).

[31] P. A. Dirac, in Proceedings of the Royal Society of London A: Mathematical, Physical and Engineering Sciences, Vol. 114 (The Royal Society, 1927) pp. 243-265.

[32] Y. P. Rakovich, F. Jäckel, J. F. Donegan, and A. L. Rogach, J. Mat. Chem. 22, 20831 (2012).

[33] X. Duan, Y. Huang, Y. Cui, J. Wang, and C. M. Lieber, Nature 409, 66 (2001).

[34] F. Frezza, L. Pajewski, D. Saccoccioni, and G. Schettini, Optics Communications 265, 47 (2006).

[35] R. Bennett, T. M. Barlow, and A. Beige, Eur. J. Phys. 37, 014001 (2015).

[36] R. El-Ganainy and S. John, New J. Phys 15, 083033 (2013).
[37] M. Abramowitz and I. A. Stegun, Handbook of mathematical functions: with formulas, graphs, and mathematical tables, 55 (Courier Corporation, New York, 1964).

[38] P. Berman, R. W. Boyd, and P. W. Milonni, Phys. Rev. A 74, 053816 (2006).

[39] P. Milonni, R. Loudon, P. R. Berman, and S. M. Barnett, Phys. Rev. A 77, 043835 (2008).

[40] I. L. Medintz and H. Mattoussi, Physical єhemistry єhemical physics 11, 17 (2009).

[41] U. Resch-Genger, M. Grabolle, S. Cavaliere-Jaricot, R. Nitschke, and T. Nann, Nature methods 5, 763 (2008).

[42] J. M. Klostranec and W. C. Chan, Advanced Materials 18, 1953 (2006).

[43] G. D. Scholes and D. L. Andrews, Phys. Rev. B 72, 125331 (2005).

[44] D. S. Bradshaw and D. L. Andrews, Superlattices and Microstructures 47, 308 (2010).

[45] D. S. Bradshaw and D. L. Andrews, The Journal of ehemical physics 128, 144506 (2008).

[46] D. L. Andrews, R. G. Crisp, and S. Li, The Journal of ehemical physics 127, 174702 (2007).

[47] D. S. Bradshaw and D. L. Andrews, Journal of Nanophotonics 3, 031503 (2009).

[48] R. D. Mattuck, A Guide to Feynman Diagrams in the Many-Body Problem (Dover, Newyork, 1992). 